\title{
Identification hydrologique des événements secs extrêmes dans le bassin d'Ichkeul (Tunisie)
}

\author{
MAJID MATHLOUTHI ${ }^{1}$ \& FETHI LEBDI ${ }^{2}$ \\ 1 Laboratoire de Recherche en Sciences et Techniques de l'Eau de l'INAT, 43 Avenue Charles Nicolle 1082 Tunis, \\ Tunisie \\ majid mathlouthi@yahoo.fr \\ 2 Institut National Agronomique de Tunisie (INAT), 43 Avenue Charles Nicolle 1082 Tunis, Tunisie - Université de Carthage
}

\section{INTRODUCTION}

Les modèles stochastiques de conditions météorologiques sont souvent utilisés en complément de données climatologiques quotidiennes observées et peuvent également être utilisés pour générer de longues séries chronologiques de données synthétiques pour l'analyse des risques. Ils mettent l'accent sur les variations jour au jour dans les paramètres météorologiques. La précipitation est un élément très important du climat qui affecte en même temps l'environnement naturel et la société humaine. Des événements allant des sécheresses prolongées au court terme, inondations de forte intensité sont souvent associés à des effets dévastateurs pour la société et l'environnement. Le phénomène de sécheresse a été étudié en fonction de la série des évènements pluvieux, par plusieurs auteurs dont nous pouvons citer Fogel et Duckstein (1982), Mathlouthi et Lebdi (2008, 2009) et Mathlouthi (2009). L'objectif principal de cet article est l'analyse par événement des périodes sèches basées sur des séries d'observations de la pluie journalière.

\section{DONNEES}

L'approche utilise les observations de la pluie journalière aux stations pluviométriques actives de 1968 à 2010 dans le bassin d'Ichkeul, au Nord de la Tunisie. La zone fait partie de la région naturelle Tellienne montagneuse froide et pluvieuse.

\section{MÉTHODOLOGIE}

Un événement est une durée dans le temps d'une pluie ou d'une sécheresse continue. Les évènements secs sont constitués d'une série de jours secs encadrés par des évènements pluvieux. Par conséquent, l'analyse par événement définit la saison humide comme étant une succession d'événements de pluie et d'événements secs. Les événements pluvieux eux-mêmes sont définis sous forme d'une série ininterrompue de jours pluvieux comprenant au moins un jour ayant reçu une précipitation supérieure à un seuil prédéterminé (Mathlouthi et Lebdi 2008, 2009). Une valeur seuil de pluie journalière de $3.6 \mathrm{~mm} \mathrm{j}^{-1}$ a été choisie.

\section{RESULTATS}

Le tableau 1 donne la durée de la période sèche mensuelle maximum. Des périodes sèches sévères de 51 et 60 jours respectivement aux mois de septembre et mars ont été observées à la station de la coopérative Bakhraya. Egalement, un extrême de 49 jours en septembre a été enregistré à la station de Frétissa SM.

Tableau 1 Durée de la période sèche mensuelle maximum (en jours).

\begin{tabular}{llllllllll}
\hline Station & Période d'observation & Sept. & Oct. & Nov. & Déc. & Jan. & Fév. & Mars & Avril \\
\hline Ghézala-Barrage & $1968 / 2010$ & 29 & 30 & 28 & 27 & 40 & 41 & 25 & 42 \\
$\begin{array}{l}\text { Coopérative } \\
\text { Bakhraya }\end{array}$ & $1969 / 2010^{*}$ & 51 & 35 & 31 & 27 & 35 & 43 & 60 & 42 \\
Frétissa SM & $1982 / 2010$ & 49 & 42 & 29 & 41 & 38 & 41 & 26 & 34 \\
Sidi Abdelbasset & $1968 / 2010^{+}$ & 36 & 45 & 22 & 29 & 46 & 41 & 32 & 24 \\
\hline
\end{tabular}

\footnotetext{
${ }^{*}$ non observée durant 12 ans; ${ }^{+}$non observée durant 14 ans.
} 
La Fig. 1 représente les effectifs observés et théoriques (distribution binomiale négative ajustée) pour la station pluviométrique de Ghézala-barrage. Elle montre que la plus courte interruption (un jour) est la plus fréquente. Presque 19\% des évènements secs ont une durée seulement d'un jour. Toutefois, le champ observé est beaucoup plus long que la durée de l'évènement de pluie. Des périodes sèches jusqu'à 30 jours et même plus peuvent être enregistrées, cependant la probabilité que de telle longueur extrême se produise au milieu de la saison humide est faible.

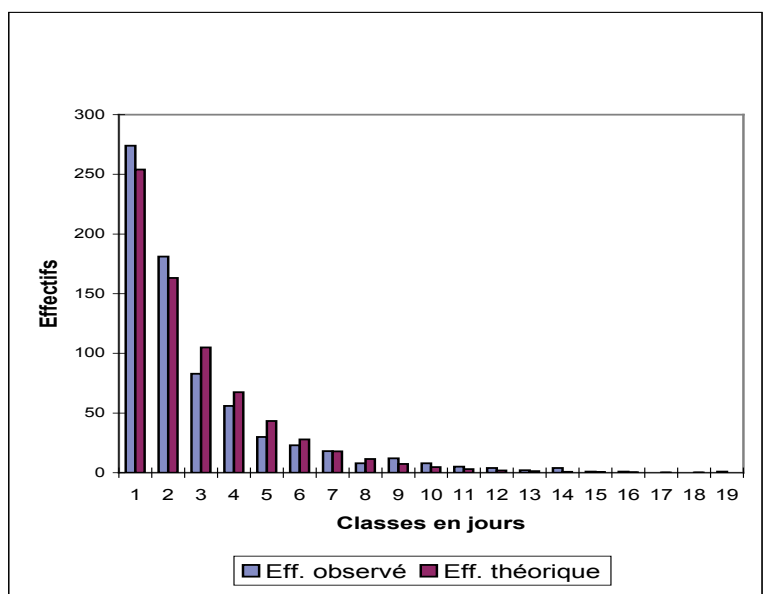

Fig. 1 Distribution de l'événement sec à la station de Ghézala-Barrage.

\section{DISCUSSION ET CONCLUSIONS}

L'analyse des événements secs faisant appel à l'hypothèse de l'indépendance des événements successifs, donne une méthode alternative pour examiner les phénomènes de sécheresse et permet de planifier les ressources hydriques sur une base différente de celle des observations faites à intervalle de temps régulier. Malheureusement la longueur des séries d'observations disponibles est souvent bien plus courte, surtout en zones semi-arides (Mathlouthi and Lebdi 2009).

\section{REFERENCES}

Fogel, M. M. and Duckstein, L. (1982) Stochastic precipitation modelling for evaluating non-point source pollution. In: Statistical Analysis of Rainfall and Runoff (Proc. Int. Symp. on Rainfall-Runoff Modelling 1981) (ed. by V. Singh), 119-136. Water Resources Publications. Littleton, Colorado, USA.

Mathlouthi, M. (2009) Optimisation des règles de gestion des barrages réservoirs pour des évènements extrêmes de sècheresse. Thèse de Doctorat, Institut National Agronomique de Tunisie, Tunis, TUNISIE.

Mathlouthi, M. and Lebdi, F. (2008) Event in the case of a single reservoir: the Ghèzala dam in Northern Tunisia. Stochast. Environ. Res. Risk Assess. 22, 513-528.

Mathlouthi, M. and Lebdi, F. (2009) Analyse statistique des séquences sèches dans un bassin du nord de la Tunisie. Hydrol. Sci. J. 54(3), 442-455. 